\title{
Synthesis and Characterization of Carbon Micro Balls from Graphite by the Arc Discharge Method
}

\author{
Arshad Hussain Wazir ${ }^{* 1}$, Abdul Manan ${ }^{2}$, Murad Khan ${ }^{2}$, Imdad Ullah Qurashi ${ }^{1}$, \\ Wahib Noor Khan ${ }^{1,2}$, Khurram Yaqoob ${ }^{3}$ and Safeer Ahmad ${ }^{4}$

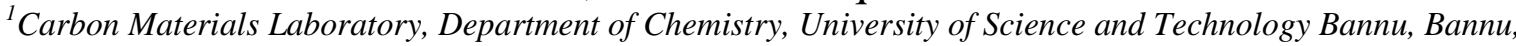 \\ Khyber Pukhtunkhwa 28100, Pakistan. \\ ${ }^{2}$ Laboratory for Research in Advanced Materials, Department of Physics, University of Science and Technology \\ Bannu, Bannu, Khyber Pakhtunkhawa 28100, Pakistan. \\ ${ }^{3}$ School of Chemical and Materials Engineering, National University of Science and Technology, \\ Islamabad, Pakistan. \\ ${ }^{4}$ Department of Physics, Islamia College, Peshawar, Khyber Pakhtunkhawa 25120, Pakistan. \\ *Corresponding Author Email: arshadwaziri@gmail.com \\ Received 02 October 2018, Revised 04 March 2019, Accepted 30 June 2019
}

\begin{abstract}
During the arc discharge technique, a high direct current (DC) voltage powers have been employed by researchers for the fabrication of carbon materials, while in this reported technique, a very low DC power is used to produce carbon micro balls (CMBs). Wherein, the CMBs were synthesized with a minimum capacity of current (1.5 amperes) and voltage (15 volts), and their structural properties were investigated. The electrochemical arc discharge was performed between two graphite rods inserted in an aqueous solution of $1 \%$ acetonitrile. The prepared CMBs were characterized using X-ray Diffraction (XRD), Scanning Electron Microscope (SEM), Attenuated Total Reflectance Fourier transform infrared spectroscopy (ATR-FTIR) and Energy Dispersive Xray $(\mathrm{EDX})$ analysis for confirmation of their characteristic properties. The existence of spherical shape and smooth surface of the CMBs was confirmed by SEM with diameters in the range of $80 \mu-300 \mathrm{~nm}$, and the EDX analysis exhibited the composition of CMBs with atomic mass percents of carbon $(88.35 \%)$ and oxygen $(11.65 \%)$. Similarly, the XRD analysis revealed the crystalline graphitic carbon nature of the CMBs with an average crystallite size calculated from the major diffraction peak using the Scherrer formula of about $40.59 \mathrm{~nm}$. The FTIR analysis also showed the signs of the aromatic and oxygen functionalities present in the CMBs.
\end{abstract}

Keywords: Arc discharge, Carbon micro balls, Scanning electron microscopy, X-ray diffraction, FTIR spectroscopy.

\section{Introduction}

The manufacture of carbon based nano-materials such as fullerenes discovered in 1985 [1], carbon nanotubes [2] in 1991, carbon nano rods, carbon nano balls (nano spheres) and graphene in 2004 [3] are of tremendous importance due to their specialized applications in broad areas of science and technology. The importance of these materials stems from their characteristic properties such as high strength, small dimensions, high electrical conductivity, high thermal conductivity and a low thermal expansion coefficient. The synthesis of these carbon products can be roughly divided into two types of methods. The first one is mainly based on the sublimation of carbon in an inert atmosphere, such as an electric arc discharge process [4], laser ablation [5] or sublimation by solar energy [6], and the second includes catalytic decomposition of suitable organic precursors [7], electrolysis in a molten ionic salt [8], heat treatment of polymers [9], low temperature solid 
pyrolysis [10] and etc. Similarly, arc discharge techniques for the synthesis of carbon materials are also applied which are considered to be more cost effective as compared to among the above mentioned processes [11], because the carbon nanospheres (nanoballs) have also received great attentions due to their potential applications in anodes for lithium-ion batteries, catalyst supports, electrodes for super capacitors, lubricants, polymers, rubber additives and etc. [12, 13]. For example, He et al. [14] investigated the growth of carbon nano balls in the presence of acetylene with coke powder as carbon source by arc discharge technique. The resulted products were characterized using Field emission scanning electron microscopy (FE-SEM), field emission scanning and transmission electron microscope (STEM) equipped with energy dispersive X-ray (EDX), X-ray diffraction (XRD), and Raman spectroscopy to confirm their characteristic properties.

Similarly, Wu et al. [15] also presented a novel synthesis of carbon nano balls with diameters in the range of 30-70 $\mathrm{nm}$ using starch as carbon source, followed by vaporization of carbohydrate by arc under a helium atmosphere and characterized the resulted product with transmission electron microscopy (TEM), EDX and XRD.

The present work also deals with the formation of $\mathrm{CMBs}$ by employing the electrochemical arc discharge technique with a low DC power of 1.5 amperes and 15 volts as compared to the previous research workers that used high DC powers to fabricate carbon materials. Therefore, the aim of this study is focused on the preparation of CMBs from the graphite anode in the arc discharge process employing low DC power. The detailed analysis of the obtained CMBs by means of FTIR, XRD, and SEM is also performed.

\section{Materials and Method}

For this work, the graphite rods $(150 \mathrm{~mm}$ in length and $3 \mathrm{~mm}$ in diameter) and acetonitrile $\left.\left(\mathrm{C}_{2} \mathrm{H}_{3} \mathrm{~N}\right)\right)$ of analytical grad reagent were purchased from Sigma-Aldrich. The DC voltage power supply (LD-Didicatic GmbH, 52145, made in Germany) with capacity of current (1.5 amperes) and voltage (0 to 15 volts) was used.

\section{The synthesis of CMBs}

For the synthesis of CMBs, a low DC voltage arc discharge technique was performed. Wherein, two graphite rods as electrodes (anode and cathode) were connected with a copper wire to the DC voltage power supply and were inserted in an electrolytic cell containing an electrolyte solution made of acetonirile in deionized water (acetonitrile is an aprotic organic solvents used in electrochemistry). The process of CMBs formation in the DC arc discharge cell is shown in Fig. 1. By switched on the DC voltage power supply, the evaporation of the graphite anode was started when the anode was touched with the cathode in pulses under in an electrolyte solution (anode was touched momentarily with the cathode to discharge the residual charge). The process was performed at various conditions of DC voltages (5, 10 and 15 volts) and various percent concentrations of an electrolyte solution in deionize water $(0.5,1$ and $1.5 \%$ ) for time interval of 30 minutes to get an optimum product yield. During the optimization process, the formation of the product yield was found to be $50 \%$ by weight with an optimum concentration of electrolyte solution of $1 \%$ acetonitrile and with a maximum voltage of 15 volts for 30 minutes. The percent yield as a function of the arc discharge process with various voltages and various electrolyte solutions for 30 minutes were also tabulated as shown in Table 1 . After completion of the process, the particles present in the precipitated and suspended forms in the electrolyte solution were filtered and washed, respectively. The residues found after filtration and washing were collected and dried in an oven at 105 ${ }^{\circ} \mathrm{C}$ and finally the $\mathrm{CMBs}$ in powder form were desiccated for further use to characterize with different techniques for confirmation of their characteristic properties. 


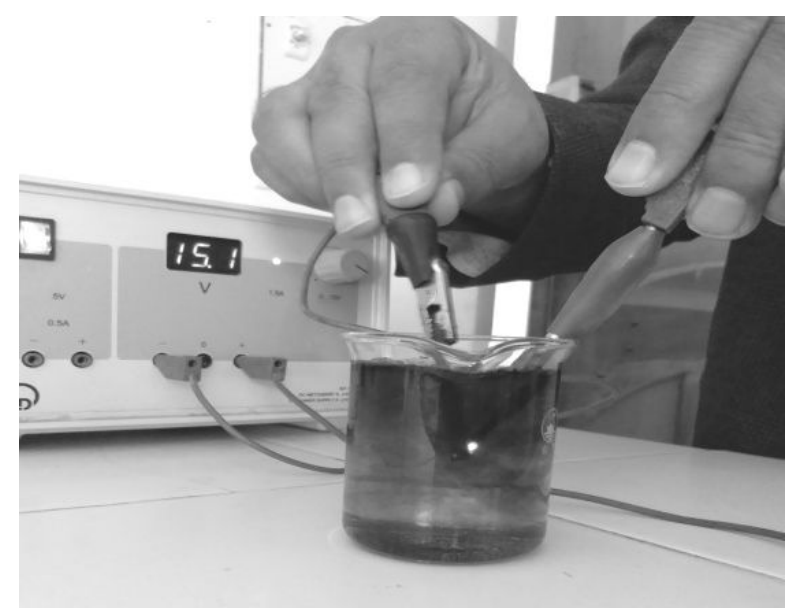

Figure 1. Process of CMBs formation using DC are discharge

Table 1. The yield (\%) as a function of arc discharge with various voltages and concentrations of electrolyte solutions for $\mathbf{3 0}$ minutes.

\begin{tabular}{cccc}
\hline $\begin{array}{c}\text { DC Voltage } \\
(\text { volts })\end{array}$ & $\begin{array}{c}\text { Time } \\
(\mathbf{m i n})\end{array}$ & $\begin{array}{c}\text { Concentration of } \\
\text { electrolyte solution } \\
(\%)\end{array}$ & $\begin{array}{c}\text { Percent } \\
\text { weight } \\
(\%)\end{array}$ \\
\hline 5 & 30 & 0.5 & 15 \\
10 & 30 & 1 & 50 \\
15 & 30 & 1.5 & 35 \\
\hline
\end{tabular}

\section{Characterization}

The surface morphology of the CMBs was examined under a Scanning Electron Microscope (Model; JSEM5910, JEOL, Japan). Similarly, the elemental analysis of the product was also performed using Energy Dispersive X-ray Spectroscopy (EDX with SEM (JEM5910) INCA200/Oxford Instruments, UK).

The CMBs in powder form were used in the X-ray diffraction (XRD) analysis. The XRD patterns of the samples were obtained by a diffractometer (JEOL X-ray diffractometer, model JDX-3532, Japan) using X-rays; CuKa $(\lambda=1.5418 \AA)$.

Scherrer equation was used to determine the crystallite size from $\mathrm{X}$-ray diffraction pattern measured for nanoparticles:

$$
\mathrm{d}=\frac{\mathrm{K} \lambda}{\beta \cos \theta}
$$

Where $\mathrm{K}$ is the Scherrer constant (shape factor, its value is 0.9 ), $\mathrm{k}$ is the $\mathrm{X}$-ray wavelength $(\lambda=0.15418 \mathrm{~nm}), \beta$ is the line broadening at half the maximum intensity (FWHM) in radians, $\theta$ is the Bragg angle, (the position of the diffraction peak maximum) and $d$ is the averaged dimension of crystallites in nanometers.

Infra-Red Spectroscopy (Eco-ATR Spectrometer, Alpha, Bruker) was also used to characterize the presence of functional groups in the CMBs.

\section{Results and Discussion Morphology and energy dispersive $x$-ray analysis of CMBs}

The SEM micro photographs (with low and high magnification) of the product obtained during electrochemical arc discharge are shown in the Fig. 2a-c. Wherein, the CMBSs with spherical shape and smooth surface can be observed. The large quantity of CMBSs with diameters in the range of $80-300 \mathrm{~nm}$ were produced as graphically shown in Fig. 2d. The EDX analysis is shown in Fig. 3 which reveals that the CMBs are composed of carbon and oxygen with atomic mass percents of 88.35 and 11.65 , respectively. The presence of oxygen atom may be due to the electrochemical oxidation of graphite or the absorption of air [16]. Thus the EDX results confirm that the carbon was the dominant element and no metals were found in the product. From the SEM images the spherical shape of carbon particles can be seen.

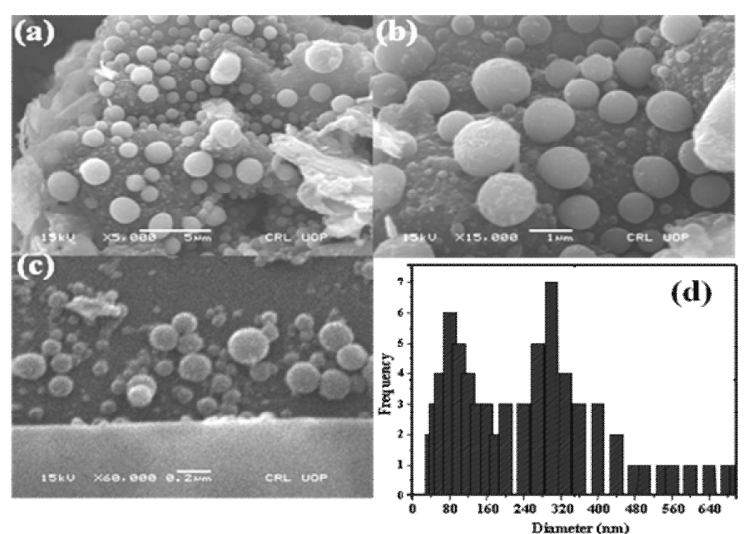

Figure 2. Surface morphology of the synthesized CMBs $(a, b)$ with low-magnification SEM image and (c) with high-magnification SEM image, and (d) graphical representation of diameters of CMBs. 


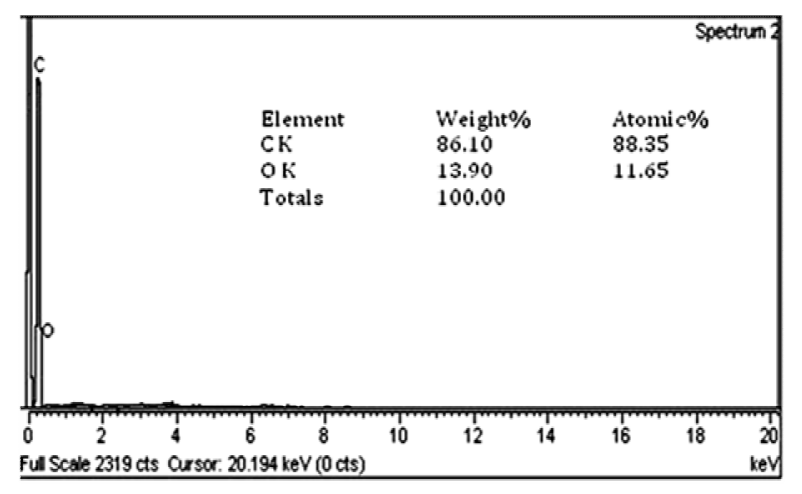

Figure 3. EDX spectrum of CMBs.

\section{X-ray diffractometry}

The XRD pattern of CMBs is shown in Fig. 4. The profile shows two peaks at $2 \theta$ of $23.85^{\circ}$ and $26.55^{\circ}$ (with interlayer spacing $3.73 \AA$ and $3.36 \AA$ calculated according to the Bragg's equation) corresponding to the amorphous and the graphitic nature of the resulted product (JCPDS Code: 00-008-0415) as also described earlier in the literature $[14,17-24]$. The mean size of the crystalline CMBs was also calculated from the major diffraction peak using the Scherrer formula which was found to be $40.59 \mathrm{~nm}$. It suggests that the prepared CMBs mostly consist of crystalline graphitic carbons and less amount of amorphous carbons.

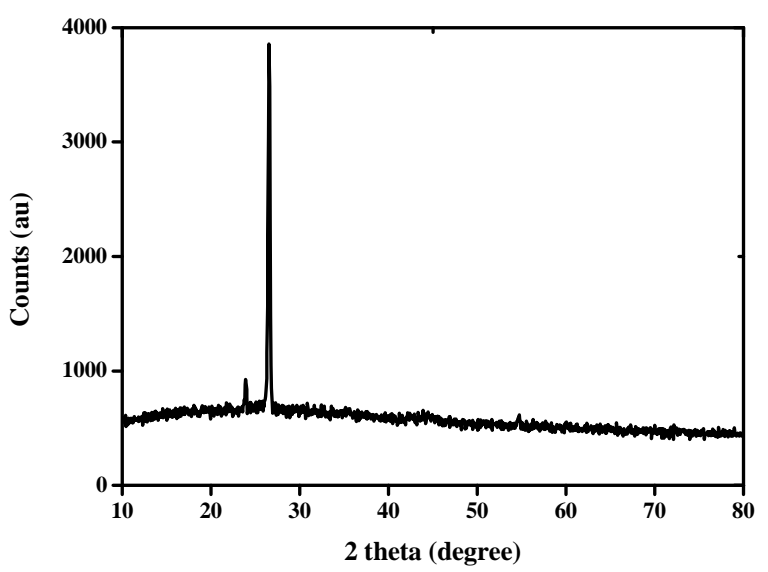

Figure 4. XRD profile of CMBs

\section{FTIR study}

The ATR-FTIR spectrum of the CMBs is shown in Fig. 5 exhibiting broad and featured signals at various wave numbers such as 3418 ,
3098, 2318, 1692, 1645 and $1514 \mathrm{~cm}^{-1}$. The absorption band observed at $3418 \mathrm{~cm}^{-1}$ is mainly due to the presence of $\mathrm{O}-\mathrm{H}$ and $\mathrm{N}-\mathrm{H}$ groups. A small peak can also be seen at wave number 3098 $\mathrm{cm}^{-1}$ which may be ascribed to the presence of aromatic ring in the CMBs. The presence of peak at $2318 \mathrm{~cm}^{-1}$ is assigned to the $\mathrm{C}=\mathrm{C}$ stretching vibration. The peaks observed at $1692 \mathrm{~cm}^{-1}$ and $1645 \mathrm{~cm}^{-1}$ correspond to the stretching vibrations of $\mathrm{C}=\mathrm{O}$ and $\mathrm{C}=\mathrm{C}$ bonds. The small peak appears at $1514 \mathrm{~cm}^{-1}$ is due to the hexagonal $\mathrm{C}=\mathrm{C}$ bond of the CMBs formed as reported earlier in the literature [14-24]. The presence of these oxygen and nitrogen functional groups on the materials can perform as active sites, chemisorbing the reactants and forming surface intermediates of sufficient strength. Because, the basic carbons are considered to be the most active for environmental catalysis applications, both in the gas and liquid phases as described earlier [25].

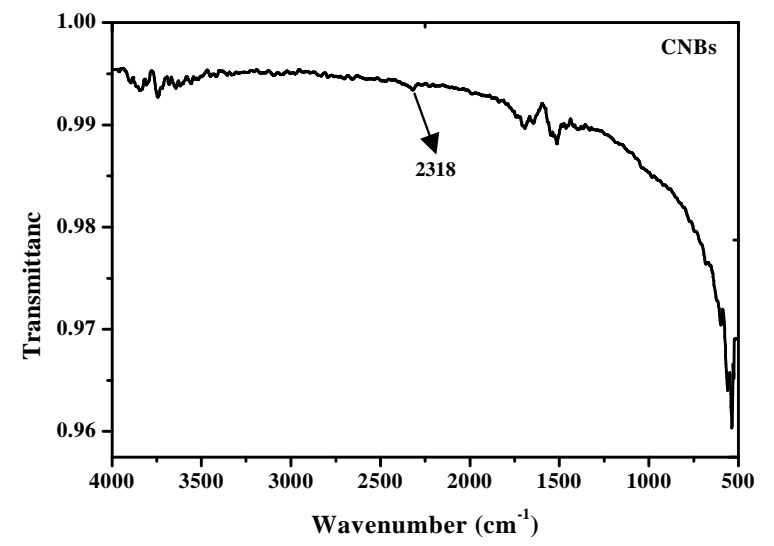

Figure 5. ATR-FTIR profile of CMBs

\section{Conclusion}

The aim of this work was to produce CMBs from graphite rod by an electrochemical arc discharge technique with low DC voltage power. The maximum product yield was found to be $50 \%$ by weight using $1 \%$ electrolyte solution of acetonitrile in deionized water with a maximum voltage of 15 volts for time duration of 30 minutes. The SEM images of the obtained product revealed that the CMBs exist with spherical shape and smooth surface. The EDX analysis showed the composition of CMBs with atomic masses of $88.35 \%$ carbon and $11.65 \%$ oxygen. Similarly, the XRD analysis confirmed the crystalline graphitic 
nature of CMBs. The FTIR analysis exhibited the presence of significant functional groups in the CMBs. The present work deals with the preparation of high purity CMBs that could be used for applications as reinforced rubber additives, lubricating materials, column packing materials, catalyst supports and for environmental catalysis applications.

\section{References}

1. H. W. Kroto, J. R. Heath, S. C. O. Brien, R. F. Curl and R. E. Smalley, Nature, 318 (1985) 162. doi: $10.1038 / 318162 \mathrm{a} 0$

2. $\quad$ S. Iijima, Nature, 354 (1991) 56. http://dx.doi.org/10.1038/354056a0

3. K. S. Novoselov, A.K. Geim, S.V. Morozov, D. Jiang, Y. Zhang, S. V. Dubonos, I. V. Grigorieva and A. A. Firsov, Science, 306 (2004) 666.

doi: $10.1126 /$ science. 1102896

4. S. Seraphi, D. Zhou and J. Jiao, J. Appl. Phys., 80 (1996) 2097. https://doi.org/10.1063/1.363102

5. B. H. Liu, J. Ding, Z. Y. Zhong, Z. L. Dong, T. White and J. Y. Lin, Chem. Phys. Lett., 358 (2002) 96. doi: 10.1016/S0009-2614(02)00592-4

6. X. C. Sun and N. Nava, Nano Lett., 2 (2002) 765.

doi: $10.1021 / \mathrm{n} 10255911$

7. E. Flahaut, F. Agnoli, J. Sloan, C. O. Connor and M. L. H. Green, Chem. Mater., 14 (2002) 2553. doi: $10.1021 / \mathrm{cm} 011287 \mathrm{~h}$

8. Y. Saito, Carbon, 33 (1995) 979. https://doi.org/10.1016/00086223(95)00026-A

9. J. Jiao, S. Seraphin, X. Wang and J. C. Withers, J. Appl. Phys., 80 (1996) 103. https://doi.org/10.1063/1.362765

10. L. D. Book, C. H. Xu and G. E. Scuseria, Chem. Phys. Lett., 222 (1994) 281. https://doi.org/10.1016/00092614(94)00341-6

11. R. Kumar, R. K. Singh, P. K. Dubey, R. M. Yadav, D. P. Singh, R. S. Tiwari and O. N. Srivastava, J. Nanopart. Res., 17 (2015) 24. doi: 10.1007/s11051-014-2837-9
12. Y. Wang, F. Su, C. D. Wood, J. Y. Lee and X. S. Zhao, Ind. Eng. Chem. Res., 47 (2008) 2294. doi: 10.1021/ie071337d

13. N. Zhao, J. Wang, C. Shi, E. Liu, J. Li and C. He, J. Alloys Compounds, 617 (2014) 816. https://doi.org/10.1016/j.jallcom.2014.08.072

14. X. He, F. Wu and M. Zheng, Diamond Related Mater., 16 (2007) 311. https://doi.org/10.1016/j.diamond.2006.06.011

15. X. J. Wu, X. Yuan and L. G. Yu, Adv. Mater. Rese., 476-478 (2012) 1533. https://doi.org/10.4028/www.scientific.net/A MR.476-478.1533

16. F. Niu, Y. Xu, M. Liu, J. Sun, P. Guo, J. Liu, Nanoscale, 8 (2016) 5470. doi: 10.1039/c6nr00023a

17. M. A. Hossain and S. Islam, A. J. Nanosci. Nanotech., 1 (2013) 52. doi: 10.11648/j.nano.20130102.12

18. J. E. Im, H. N. Lee, J. Li and Y. R. Kim, Bull. Korean Chem. Soc., 35 (2014) 871. http://dx.doi.org/10.5012/bkcs.2014.35.3.871

19. C. Zhang, G. Bhargava, M. D. Elwell, S. Parasher, B. Zhou, D.Yates, I. Knoke, I. Neitzel and Y. Gogotsi, J. Mater. Sci., 49 (2014) 1947. doi: 10.1007/s10853-013-7796-5

20. H. C. Wu, C. T. Hong, H. T. Chiu and Y. Y. Li, Diamond Related Mater., 18 (2009) 601. https://doi.org/10.1016/j.diamond.2008.10.047

21. A. K. Pal, R. K. Roy, S. K. Mandal, S. Gupta and B. Deb, Thin Solid Films, 476 (2005) 288.

https://doi.org/10.1016/j.tsf.2004.09.064

22. T. Das, B. K. Saikia and B. P. Baruah, Gondwana Res., 31 (2016) 295.

https://doi.org/10.1016/j.gr.2015.01.012

23. O. O. Sonibare, T. Haeger and S. F. Foley, Energy, 35 (2010) 5347. https://doi.org/10.1016/j.energy.2010.07.025

24. J. Shu, R. Ma, M. Shui, D. Wang, N. Long, Y. Ren, R. Zhang, J. Yao, X. Mao, W. Zheng and S. Gao, RSC Adv., 2 (2012) 5806. doi: $10.1039 / \mathrm{C} 2 \mathrm{RA} 20284 \mathrm{~K}$

25. J. L Figueiredo and M. F. R. Pereira. Catalysis Today, 150 (2010) 2. doi:10.1016/j.cattod.2009.04.010 